\title{
Comparing TCV experimental VDE responses with DINA code simulations
}

\author{
J-Y Favez ${ }^{\dagger}$, R R Khayrutdinov ${ }^{\ddagger}$, J B Lister ${ }^{\dagger}$ and V E Lukash ${ }^{\S}$ \\ $\dagger$ Centre de Recherches en Physique des Plasmas, Association \\ EURATOM-Confédération Suisse, EPFL, 1015 Lausanne, Switzerland \\ $\ddagger$ TRINITI, Troitsk, Russia \\ $\S$ RRC Kurchatov Institute, Moscow, Russia \\ E-mail: jean-yves.favez@epfl.ch, jo.lister@epfl.ch
}

\begin{abstract}
The DINA free-boundary equilibrium simulation code has been implemented for TCV, including the full TCV feedback and diagnostic systems. First results showed good agreement with control coil perturbations and correctly reproduced certain non-linear features in the experimental measurements. The latest DINA code simulations, presented in this paper, exploit discharges with different crosssectional shapes and different vertical instability growth rates which were subjected to controlled Vertical Displacement Events, extending previous work with the DINA code on the DIII-D tokamak. The height of the TCV vessel allows observation of the nonlinear evolution of the VDE growth rate as regions of different vertical field decay index are crossed. The vertical movement of the plasma is found to be well modeled. For most experiments, DINA reproduces the S-shape of the vertical displacement in TCV with excellent precision. This behaviour cannot be modeled using linear time-independent models because of the predominant exponential shape due to the unstable pole of any linear time-independent model. The other most common equilibrium parameters like the plasma current $I_{p}$, the elongation $\kappa$, the triangularity $\delta$, the safety factor $q$, the ratio between the averaged plasma kinetic pressure and the pressure of the poloidal magnetic field at the edge of the plasma $\beta_{p}$ and the internal self inductance $l_{i}$ also show acceptable agreement. The evolution of the growth rate $\gamma$ is estimated and compared with the evolution of the closed loop growth rate calculated with the RZIP linear model, confirming the origin of the observed behaviour.
\end{abstract}

Submitted to: Plasma Phys. Control. Fusion

\section{Introduction}

A non-linear time-evolution code is an essential tool for modelling existing or future tokamak experiments in particular cases in which linearised models are inadequate. Such cases are dominated by those in which the excursions from the nominal equilibrium are large, excluding local linearisation of the plasma equilibrium response.

The DINA code [1] is a suitable code for such purposes. It assumes zero plasma mass, eliminating the dilemma introduced by falsifying the plasma mass, typified by the TSC 
code [2]. It correctly treats the poloidal flux diffusion, avoiding the imposition of either a rigid current displacement, typified by the RZIP model [3], or the frequent assumption of constant normalised poloidal flux profiles but deformable equilibrium $[4,5]$. For the work in this paper, DINA uses a simplified energy transport model and a simple sawtoothing model. For all these reasons, DINA provides a suitable compromise for plasma equilibrium response modelling.

In previous work [6] DINA showed good agreement with the effects of Poloidal Field (PF) control coil voltage pulses in TCV. A non-linear response due to one of the perturbations significantly changing the vertical field decay index and driving the closed loop unstable gave a first demonstration of non-linear behaviour correctly modelled by DINA, including the full TCV feedback and diagnostic systems. DINA has also been used in the past to study Vertical Displacement Events in the DIII-D tokamak [7].

The latest DINA code simulations, presented in this paper, exploit discharges with different cross-sectional shapes and different vertical instability growth rates which were subjected to controlled VDEs. We exploit the large height of the TCV vacuum vessel to explore a second non-linearity, namely one in which the spatial variation of the vacuum field is large enough to invalidate local linearisation of the plasma equilibrium response. A set of experiments carried out earlier to compare experimental and modelled growth rates [8] provided suitable data. Subsequently similar experiments at higher growth rate were performed, but are not analysed here [9]. In the previous work, the initial, small amplitude, growth rate was evaluated. We extend that work by reproducing the behaviour during the complete large vertical plasma displacement. This case cannot be modeled with a linear model because of the large amplitude non-linearities due to the final large vertical position displacement and non-time-invariant non-linearities since the growth rate $\gamma$ changes as a function of the position and therefore of time. The DINA code has been cross-checked against all 14 of the VDE experiments with different growth rates ranging approximately from $100 \mathrm{rad} / \mathrm{s}$ to $300 \mathrm{rad} / \mathrm{s}$ and different plasma shapes $(\delta)$ carried out on limited plasmas in the TCV tokamak.

The remainder of the paper is laid out as follows. In section 2 we describe the procedure that we used to initiate the VDE and discuss the potential disagreements which can arise when comparing the results between LIUQE and DINA simulations. In section 3 we present a comparison of the DINA simulation data with experimental data from the TCV tokamak focusing on the VDE experiments. We also present a comparison of common equilibrium parameters like the plasma current $I_{p}$, the elongation $\kappa$, the triangularity $\delta$, the safety factor $q$, the ratio between the averaged plasma kinetic pressure and the pressure of the poloidal magnetic field at the edge of the plasma $\beta_{p}$ and the internal self inductance $l_{i}$. Section 4 deals specifically with the non-time-invariant growth rate $\gamma$. We compare the time-varying growth rate of the TCV experiment and DINA simulation with the evolution of the closed-loop growth rate calculated with the RZIP model at each equilibrium point. Section 5 closes the paper with a discussion. 


\section{Experimental conditions}

\subsection{Initiating the $V D E$}

In the TCV experiments the VDE was initiated by interrupting the feedback control of the radial and vertical position at $t=0.45 \mathrm{~s}$. Because of the plasma disturbances and noise, mainly generated by the power supply and the diagnostics, and due to the fact that the plasma is unstable in vertical direction, the VDE is immediately initiated. In a DINA simulation, a break off of the control does not cause a VDE due to the fact that the plasma is in equilibrium and no noise and disturbances are added to the simulation. Therefore, a disturbance had to be injected to cause a loss of equilibrium and a subsequent VDE. Several trials had to be undertaken to find out which disturbance least pollutes the results. For the first tests we applied a trapezoidal feedforward voltage disturbance at $t=0.45 \mathrm{~s}$ during $t_{p}=4 \mathrm{~ms}$ to the E1 coil (figure 1). The sign and amplitude of the disturbance voltage determine the direction of the vertical displacement: upwards or downwards. It was found that the amplitude has to be huge $(1000 \mathrm{~V})$ to make sure that the displacement goes in the same direction as the experiment. In addition to this problem, the disturbance generates an overshoot or undershoot of the plasma current, according to the displacement direction and thus to the sign of the voltage. This overshoot or undershoot can be explained by the net flux induced by the perturbation. An $I_{p}$ overshoot is illustrated in figure 2 (top). To avoid this problem we used an antisymmetric disturbance on the E1 and E8 coils (figure 1) with the amplitude as small as possible and a period of only $t_{p}=2 \mathrm{~ms}$. As shown on figure 2 (bottom), this solution clearly prevents the overshoot of the plasma current because of the vertical anti-symmetry of the perturbation. Figure 3 shows the trapezoidal disturbance voltage of $+200 \mathrm{~V}$ and a period of $t_{p}=2 \mathrm{~ms}$ applied to coil E1 to produce an upward plasma displacement. The injected voltage in coil E8 has the same disturbance shape, but, since the two coils are driven antisymmetrically, it has a negative amplitude of $-200 \mathrm{~V}$. After this testing, this disturbance was subsequently applied for all discharges, except for discharges \#9486 and \#9490, in which the right movement direction could only be obtained with a longer $\left(t_{p}=4 \mathrm{~ms}\right)$ and bigger disturbance.

\subsection{Differences between the plasma equilibrium reconstructing code LIUQE and the simulation code DINA responses}

Before comparing the TCV experiment results (LIUQE) and the DINA simulation predictions it is necessary to consider possible discrepancies. There are several potential reasons for disagreement between the responses represented by the experimental results and the DINA simulations.

(i) Only the vertical and horizontal feedback control loops are interrupted during the VDE. The evolution of the PF currents remains controlled by other loops and determines the evolution of the vacuum field during the complete VDE. The 
agreement between the DINA and experimental evolution confirmed the correct modelling of these other feedback loops.

(ii) The electronics, particularly the amplifiers and integrators implemented in the diagnostics to evaluate the magnetic fields and the fluxes, may have small offsets. This adds offsets and constant slope drift errors to the measurements of the fields and fluxes. The power supply and electronics modelling are not considered to be a problem due to the precise agreement obtained for the linear response in previous work on TCV [6].

(iii) When starting the simulations with badly chosen initial states, a significant transient is observed before the simulation settled down to reproduce the TCV time traces more or less accurately [6]. This transient can be due to the different equilibrium parametrisation used by the LIUQE inverse equilibrium code and the DINA simulation code. However, it is difficult to find the right initial conditions to completely avoid this effect and there often remains a short transient before the equilibrium relaxes to a diffused DINA equilibrium. This can lead to an offset between the responses of the experiment and the DINA simulation. A comparison between DINA and LIUQE reconstruction of $z, \beta_{p}$ and $l_{i}$ for discharge \#9480 is shown in figure 5 . There is a clear transient for all 3 parameters at the beginning of the DINA simulation. Furthermore, after the transient has decayed, an offset error remains at a level of about $5 \%$ for $l_{i}$ and $1 \%$ for $z$. However, there is no offset error for $\beta_{p}$.

(iv) The equilibrium parameters (vertical and horizontal plasma position $\mathrm{z}$ and $\mathrm{R}$, plasma current $I_{p}$, elongation $\kappa$, triangularity $\delta$, the safety factor $q, \beta_{p}$ and $l_{i}$ ) are not measured in a direct manner. They have to be estimated offline using the accessible measurements of the diagnostics (magnetic fields, fluxes and coil currents). The LIUQE code reconstructs the plasma evolution of a discharge by means of a parameterised function set for current and pressure profiles and a fitting algorithm calculating the parameter-values of these functions (constraints). This reconstruction method leads to results that mainly follow the experiment but cannot show all details of the plasma evolution. On the other hand, the simulation with the DINA code starts with a set of initial conditions and the profiles then evolve in a totally free manner. This leads to very detailed results but, of course, only for the features of the tokamak implemented in the DINA code. Figure 4 illustrates, as an example of the potential drawback of LIUQE using parameterised functions, the evolution of the plasma current profile across the plasma mid-plane. We clearly see the smooth gaussian-like shape of the LIUQE result due to the usage of parameterised functions, while the DINA simulation shows a more complex shape evolution. Without additional experimental information we cannot determine whether the constrained or evolving profile is closer to the experimental reality. The finer structure is not 'visible' to the magnetics diagnostics outside the plasma which can measure the centroid, the total current and (less precisely) the internal 
inductance which varies with the profile width.

These problems have to be considered apart of the validation of the DINA code. Thus, a careful interpretation and treatment of the data are required to compare the results rigorously. Despite these numerous potential sources of error, the equilibrium parameters still show excellent agreement between DINA simulations and experiments, as we will see later. Additionally, two data treatment methods are adopted in order to avoid the residual offsets and drift errors. Firstly, when comparing raw diagnostic data such as magnetic fluxes and fields, the results are filtered by removing the linear part of the data, referred to as 'detrending'. Secondly, for the equilibrium parameters, and especially for the vertical position, where we have only constant offsets, a more complete data adjustement method is proposed in section 3.1.

Comparison during the final disruption itself are not as good. The most likely explanation is due to the LIUQE code which cannot deal with the significant profile evolution during disruptions without additional care. The comparison has therefore been limited to the time between the onset of the VDE up to the time just before the disruption occurs. The validation of DINA and LIUQE during the disruptive phase of TCV discharges will have to be treated in future work.

\section{Comparison between TCV and DINA}

\subsection{Elimination of the offsets between experiment and DINA data}

As mentioned in section 2.2, there may be offset errors due to electronic offsets and the choice of the initial conditions. There is another mismatch shown up by the observation that the vertical plasma displacement of the DINA simulation usually begins earlier than the experimental data. In fact, the time of the beginning of the displacement is quite sensitive to the amplitude of the disturbance voltage applied to the coils E1 and E8 in the simulation (section 2.1). Several DINA simulations with different disturbance amplitudes have shown that a bigger amplitude leads to an earlier vertical displacement as expected. As mentioned above (section 2.1), we have to apply a disturbance with an amplitude that is large enough to ensure the correct direction, upwards or downwards, of the vertical plasma movement. This disturbance is bigger than the disturbances and noise of the experiment, leading to the observation that the vertical plasma displacement of DINA usually starts earlier than the one in the experiment. Before the comparison between the experimental and the DINA simulation data can be made, this time mismatch has to be corrected by means of data shifting. We chose to shift the DINA vertical position data by minimizing the cost function $\mathrm{Q}$ :

$$
\min _{\delta t, \delta z} Q=\int_{t_{1}}^{t_{2}}\left\{\min _{t_{D}}\left[w_{t}\left(t_{D}+\delta t-t_{T}\right)^{2}+w_{z}\left(z_{D}\left(t_{D}\right)+\delta z-z_{T}\left(t_{T}\right)\right)^{2}\right]\right\} d t_{T}(1)
$$

Where $\delta t$ is the shifting of the time axis, $\delta z$ the shifting of the $z$ axis (vertical position), $t_{D}$ and $z_{D}$ are the time and vertical position of the DINA simulation and $t_{T}$ and $z_{T}$ are the time and vertical position of the TCV experiment. The integral interval is defined by 
$\left[t_{1}, t_{2}\right]=\left[0.35 s, t_{T_{\text {end }}}\right]$, where $t_{T_{\text {end }}}$ is the time of the last TCV experiment sample. The two weights $w_{t}$ and $w_{z}$ were chosen to attach more importance to the fitting accuracy of either the time axis by increasing $w_{t}$ or the $z$ axis by increasing $w_{z}$. Values of $\omega_{t}=1 \mathrm{~s}^{-2}$ and $\omega_{z}=4 \cdot 10^{-4} \mathrm{~m}^{-2}$ are suitable. The minimizations in the cases studied result values in the range of $\{-3.3 \ldots 22.1\} \mathrm{ms}$ for $\delta t$ and $\{-1.75 \ldots 6.64\} \mathrm{cm}$ for $\delta z$. Only a single case exceeded $\delta z=4.5 \mathrm{~cm}$ and only the data are modified slightly, not the simulation itself.

\subsection{Comparison of the vertical plasma position}

Figure 6 shows the modelled and experimental evolution of the vertical plasma displacement of the whole set of 14 experiments with the simplest data mismatches between the DINA simulations and the experiments eliminated in the manner described. The most immediately striking feature is that all experiments with the plasma position going downwards have an S-like shape consisting of a fast exponential-like movement at the beginning and a slowing down part at the end just before the disruption occurs. Since the $z$ position at equilibrium is located at $+20 \mathrm{~cm}$, the distance the plasma can move before the disruption is shorter for the experiments with an upward going vertical displacement. Therefore only these experiments show the initial exponential-like shape. The correct reproduction of the S-like shape by DINA is an important non-linear code feature. Such behaviour cannot be modelled by linear time-independent models because of the predominant exponential shape of the vertical instability. These models only have one unstable pole corresponding to the vertical instability and although the set of stable poles could dominate the response during transient behaviour, the unstable pole and its residue have to dominate the behaviour at longer times.

In general, especially considering the potential differences between the LIUQE and DINA codes already described, we consider the overall agreement for the evolution of the vertical plasma position as excellent. Nevertheless, to attempt a more rigorous assessment, we try to distinguish between good and less good agreement (table 1). The curvature of the exponential part (knee) of the vertical position is not always exactly reproduced by DINA, shown by a double crossing of the two traces. This curvature error seems to have a systematic property, because the DINA simulations show for every upward going displacement a softer knee than the experiments (e.g. \#9478), while for the downward going movement we see a harder knee for DINA (e.g. \#9486).

Table 1. Good and less good agreement between the experiment and DINA.

\begin{tabular}{lllllllll}
\hline Good agreement & 9477 & 9480 & 9482 & 9483 & 9487 & 9491 & 9492 & 9493 \\
Less good agreement & 9478 & 9481 & 9486 & 9488 & 9490 & 9496 & & \\
\hline
\end{tabular}

\subsection{Comparison of the equilibrium parameters}

In this section, we present a comparison of the most common equilibrium parameters. Figures 7 a) - c) show the evolution of the plasma current $I_{p}$, the elongation $\kappa_{95}$, the 
triangularity $\delta_{95}$ and the safety factor $q_{95}$, the last three parameters estimated at $95 \%$ flux surface. For completeness, the plasma vertical position $z$ is added in the top row. As mentioned, we limit the comparison to the time before the final disruption occurs. To distinguish the instant of the final disruption we used the $H_{\alpha}$ signal, shown in the bottom row. The time when the disruption occurs is approximately determined by the maximum value of $H_{\alpha}$, marked by a vertical line in the other plots. The time axis of the DINA data is shifted by $\delta t$, as described in section 3.1, for all parameters. No shifting has been applied to the vertical axes.

For some discharges (\#9477, \#9478, \#9480, \#9481, \#9483, \#9488, \#9491 and \#9492), we observe a delay between DINA and LIUQE for some equilibrium parameters (an example is the plasma current) even after aligning the evolution of $\mathrm{z}$ as described. This may be due to the LIUQE code having difficulties reconstructing the plasma evolution correctly while the plasma current is rapidly decreasing and is not significant. Despite the fact that for discharge \#9491 a short and low amplitude disturbance was applied, there remains an overshoot. This overshoot seems to also affect $\kappa, \delta$ and $q$.

Plasma current $\mathbf{I}_{\mathbf{p}}$ : The agreement of the plasma current is good up to the time at which the final disruption occurs. The under- and overshoots of the DINA plasma current of discharge \#9486 and \#9490 are obvious because the initiating VDE disturbance is longer and bigger.

Elongation $\kappa_{\mathbf{9 5}}$ : For the TCV experiments, we observe a peak in $\kappa$ when the plasma current decreases slowly (discharges \#9477, \#9480, \#9482, \#9487 and \#9493). DINA reproduces this peak only for discharges \#9477 and \#9480. This is the most important disagreement and has not yet been explained. The most probable cause is differing evolutions of the LIUQE and DINA current profiles. The offset, which can be noticed for almost all discharges, is typically due to the problem of determining the right initial conditions for the simulations.

Triangularity $\delta_{\mathbf{9 5}}$ : The agreement of the triangularity is excellent for all discharges, except for discharges \#9477 and \#9478. Despite the fact that the initial values start at $t=0.3 \mathrm{~s}$ with approximately the same values as the experiments for these discharges, the system relaxes to a DINA equilibrium, which induces a significant offset of $\delta_{95}$. Like for $\kappa_{95}$, this also belongs to the problem of determining the right initial conditions for the simulations.

Safety factor $\mathbf{q}_{95}$ : The safety factor inevitably behaves like the elongation, but with a less accentuated peak amplitude.

$\beta_{\mathbf{p}}$ and $\mathbf{l}_{\mathbf{i}}$ : The two parameters $\beta_{p}$ and $l_{i}$ are shown in figure 8 . Besides the offset errors, we establish that the decreasing behaviour of $l_{i}$ is reproduced by DINA for all discharges, while for many discharges the direction of the change of $\beta_{p}$ does not agree. 
This disagreement is most likely to be attributable to the LIUQE profiles, because it cannot accurately separate $l_{i}$ and $\beta_{p}$ for these modest elongations. To illustrate this problem, we compare the sum $\beta_{p}+\frac{l_{i}}{2}$ in figure 9 , which shows that the direction of the change is correct for all experiments.

\section{Comparison of the growth rate evolution}

In this section, we compare the growth rate evolution of the TCV experiments, the DINA simulations and the simple RZIP linear model. The latter is based on the assumption of rigid displacement of the current distribution [3].

\subsection{Computing the growth rate}

The growth rates are established in RZIP by calculating the eigenvalues of the RZIP linear model in closed loop (with all feedback loops closed except for the vertical and radial positions). For the TCV experiment and the DINA simulations, the growth rates are calculated using the vertical plasma position data $z(t)$. The estimation is based on the assumption that $z(t)$ can be modeled by the function

$$
z(t)=z_{0}+\delta z e^{\int_{t_{0}}^{t} \gamma(\tau) d \tau}
$$

which is the solution of the first order differential equation

$$
\dot{z}=\frac{d z}{d t}=\gamma(t) \delta z e^{\int_{t_{0}}^{t} \gamma(\tau) d \tau}=\gamma(t)\left(z-z_{0}\right)
$$

where we assume that $z_{0}$ and $\delta z$ are constant in time and the growth rate $\gamma$ is a function of time. The time $t_{0}$ is the time at which the VDE is initiated, in our case $t_{0}=0.45 \mathrm{~s}$, with a displacement $\delta z$ from an equilibrium with zero passive structure currents.

If we assume that $\gamma$ is time-independent, the exponent in equation (2) is replaced by $\gamma t$. This gives us the classic exponentially shaped response of a linear time-independent model. The aim of this section is to show that we can reproduce the non-linear S-shape of the vertical displacement by taking this linear model and, additionally, assuming that only $\gamma$ is a function of time. This assumption allows us to estimate the growth rate simply from the evolution of the vertical plasma position. We tested the following two different methods to compute $\gamma(t)$ :

\section{- Logarithm method}

First, the offset $z_{0}$ has to be subtracted from $z(t)$

$$
\tilde{z}(t)=z(t)-z_{0}=\delta z e^{\int_{t_{0}}^{t} \gamma(\tau) d \tau}
$$

The logarithm of $\tilde{z}$ gives

$$
\ln |\tilde{z}(t)|=\ln |\delta z|+\int_{t_{0}}^{t} \gamma(\tau) d \tau
$$

We took the norm 1 of $\tilde{z}$ to avoid a logarithm of a negative value due to any noise and the arbitrary sign of the excursion. Finally, we obtain

$$
\frac{d \ln |\tilde{z}|}{d t}=\gamma(t)
$$




\section{- Quotient method}

This method simply computes the growth rate by dividing equation (3) by equation (4):

$$
\frac{\dot{z}}{\tilde{z}}=\gamma(t)
$$

The first derivative is used in both these methods and noise in the data, especially the experimental data, is amplified. We therefore filter the vertical position data of both the DINA simulation and LIUQE reconstruction by means of a second order Butterworth filter with a cut-off frequency of $\omega_{N} / 5$, where $\omega_{N}$ is the Nyquist frequency for the sampling period of the DINA data of $100 \mu \mathrm{s}$. Since LIUQE does not output data with a constant sampling rate, its data was interpolated linearly with the same sampling period as DINA and then filtered similarly.

In practice, the implementation of both methods exposed two problems:

(i) The constant values $z_{0}$ and $\delta z$ have to be determined. In fact, only one of these values has to be estimated because equation (4) evaluated at $t=t_{0}$ simplifies to

$$
z\left(t_{0}\right)=z_{0}+\delta z
$$

where $z$ is the known vertical position of either the DINA simulation or the LIUQE reconstruction data. The estimation is obtained by assuming that just after $t=t_{0}$ the growth rate $\gamma(t)$ remains time-independent over an interval defined by $\left\{t_{0} \ldots t_{l i n}\right\}$. This simplifies equation (5) to

$$
\ln \left|z(t)-z_{0}\right|=\ln |\delta z|+\gamma t
$$

In this case $\ln \left|z(t)-z_{0}\right|$ is linear and we simply tuned $\delta z$ by hand until $\ln \left|z(t)-z_{0}\right|$ showed the expected linear behaviour in its initial phase defined by $\left\{t_{0} \ldots t_{\text {lin }}\right\}$.

(ii) In principle, $z(t)-z_{0}$ should always remain positive or negative since $\delta z$ is always positive for upward going VDEs and negative for downward going VDEs. In practice, there is noise in the $z(t)$ data and therefore, at the beginning where the vertical position is close to its initial value $z\left(t_{0}\right)$, the expression $z(t)-z_{0}$ oscillates around zero, which implies a number of zero crossings. To avoid zero crossings we add or subtract, according to the displacement direction of $z$, a small positive value to $z(t)-z_{0}$. Despite this precaution, a rapidly changing $\gamma$ can never be completely avoided when noise is polluting the $z$ data. Fortunately, we are mostly interested in the results of the non-linear domain where $\gamma$ varies in time and the distance $\left|z(t)-z_{0}\right|$ is large, under which condition the noise is no longer predominant.

For what follows, we only used the logarithm method, as it was found to provide less noise-polluted results than the quotient method.

\subsection{Comparison of the growth rate}

The comparison of the growth rate is shown in figures $10 \mathrm{a}$ ) - c). The first and second rows illustrate $z(t)-z_{0}$ and $\ln \left|z(t)-z_{0}\right|$ of the LIQUE and the DINA data. The results 
of the second row can be used to find an appropriate value for $\delta z$ and $z_{0}$, as described in point i) of section 4.1. The third row shows the evaluated growth rates for LIUQE, DINA and RZIP. As mentioned in point ii) of section 4.1, we clearly see, especially for LIQUE, the noisy results in the initial phase where the growth rate is assumed to be constant. By inspecting the RZIP growth rate, we see that the assumption of a constant $\gamma$ in the initial phase appears valid.

The RZIP model uses the reconstructed equilibrium and the instantaneous values of the PF coil currents to determine the growth rate. The vacuum poloidal field in the RZIP calculation therefore varies somewhat, but the height of the $B_{R}=0$ point moves by a few $\mathrm{cm}$, since the vertical position control is switched off. It is therefore not surprising that the modelled growth rate tracks the experimentally estimated evolution of the growth rate. On the other hand, the DINA code simulates the full evolution and the growth rate evaluated from the DINA results corresponds to the growth rate given the instantaneous PF coil currents in the DINA simulation and the instantaneous position of the plasma in the simulation. If the plasma evolution $z(t)$ is different, then the DINA simulation is at a different location at a given time. Since the growth rate itself is shown to be a function of the equilibrium position, then once there is any disagreement in $z(t)$, a disagreement in the estimated growth rate is inevitable for the remainder of the discharge. In spite of this, the ability of DINA to predict the evolution of all these plasma parameters during the large scale VDE is convincing.

Three candidate explanations are proposed for such a strong variation of the growth rate.

(i) The proximity of the vacuum vessel wall.

The slowing down of the vertical displacement at the end of the downward going discharges could be attributable to the stabilising effect of the varying proximity of the vacuum vessel top and bottom wall.

(ii) Variations in the plasma current profile.

The effect on the growth rate of a varying plasma current profile is shown in figure 11. The evolution of the RZIP growth rate assuming a fixed current profile is different from the 'normal' RZIP growth rate taking the deforming reconstructed current profile. The fixed current profile is taken before the break off of the vertical and radial position feedback control at $t=0.43 \mathrm{~s}$. We previously established that the plasma current profile is not changing while the feedback control of the vertical and radial positions is active. After opening the position feedback control the deforming current profile growth rate peaks, while the fixed current profile growth rate already starts to decrease. This illustrates that the variation of the plasma current profile has a detectable impact on the evolution of the growth rate and therefore on the consequent vertical plasma displacement. Since the plasma current profiles of LIUQE and DINA are significantly different, figure 4, it is natural that the agreement between the experiments and the DINA simulations is not perfect. Moreover, by inspecting the peaks of the RZIP growth rate in figures 10 a) - c) 
we detect a systematic behaviour that discharges with large peaks (e.g. \#9477, \#9486, \#9490 and \#9496) show a less good agreement of the vertical movement. We also notice that the plasma current profile has some impact on the decrease of the growth rate at the end of the discharge, just before the disruption occurs. The deformable current profile growth rate shows a more pronounced rate of decrease.

(iii) The spatial variation of the vertical field decay index $n$.

The local vertical field decay index is

$$
n=-\frac{R}{B_{z}} \frac{\partial B_{z}}{\partial R}
$$

where $B_{z}$ is the local vertical magnetic field and $R$ is the local major radius. Figure 12 shows the spatial variation of the vacuum poloidal flux for discharge \#9487 and does not vary significantly during the VDE since the poloidal field coil currents themselves do not vary significantly. The inhomogenity of the decay index is exceptional in TCV due to the large number of poloidal field coils allowing highly structured vacuum fields and to the elongation of the vacuum vessel.

The spatial effect of the varying plasma current profile is the smallest of these effects and the characteristic behaviour must reside in the first and third effects. To illustrate the origin of the pronounced S-curve in the downward moving VDEs, figure 13 shows the stabilising and destabilising forces per unit displacement on the plasma during the VDE. The variation in the stabilising forces is due to the variation of the coupling of the plasma current distribution to the passive and active circuits (vessel and PF coils) as the plasma moves. The destabilising force per unit displacement is simply due to the convolution of the plasma current distribution with the decay index distribution, deducible from figure 12. In the case of the upward moving VDE, the stabilising force per unit displacement is reinforced almost immediately as the plasma current approaches the top wall of the vacuum vessel, while the destabilising force shows only a slight variation. In the case of the downward moving VDE, the stabilising force is slightly reduced as the plasma moves towards the mid-plane and only increases at the end of the trajectory when it couples more strongly to the lower vessel wall. During the downward moving disruption, the plasma feels an almost constant stabilising force, whereas the destabilising force varies significantly as different decay index regions are crossed. We can therefore predominantly attribute the pronounced S-curve in TCV downward moving VDEs to the spatial variation in the decay index rather than to the variation of the coupling to the vacuum vessel. TCV therefore differs from other devices in this respect, partly due to the large vertical height which reduces the sensitivity of the restoring force to plasma displacements compared with other tokamaks which have a more conformal vacuum vessel and partly due to the flexibility of its poloidal field coil system. A supporting confirmation comes from the elongation of the reconstructed plasma equilibrium, which drops to nearly 1.0 before disrupting, in the downward moving VDE. 


\section{Conclusion}

In this paper we have only had to make minor adjustments to the initial conditions of fully non-linear DINA simulations of VDEs in TCV in order to obtain convincing agreement between the experimental and modelled data. The large number of TCV discharges modelled cover a range of triangularities and growth rates. The highly elongated TCV vacuum vessel has allowed us to follow VDEs over a large distance before a disruption ensues. The vacuum field curvature varies significantly over these large distances, implying a large modification of the vertical instability growth rate during the VDE. This feature is correctly modelled by DINA and the underlying reasons are brought out by inspecting the growth rate estimated by the RZIP rigid current displacement model which is accurate for the equilibria investigated. The presence of both upward and downward going disruptions shows that the proximity to the top and the bottom of the vessel is not the determining factor for varying growth rates. The evolution of the elongation and triangularity in DINA does not agree perfectly with the LIUQE reconstructions and the multiple potential reasons are presented. Given these differences, it is impressive that the comparison shows such similar features and indeed, the inevitable differences illustrate the potential dangers of assuming absolute precision in non-linear simulations of such a complex system. However, since the role of the complete plasma control feedback system is to hide such differences, the closed loop simulations are far more accurate than the "free fall" VDE simulations.

\section{Acknowledgments}

Benchmarking these TCV experiments against the DINA code was first suggested at an ITER Expert Group meeting on MHD, Control and Disruptions. Thanks go to the TCV team without whom the experimental results would not be comparable with the model. Two of the authors (RK, VL) thank the CRPP for their hospitality during visits to Lausanne. The authors thank F. Hofmann for his comments on the manuscript. This work was partly supported by the Swiss National Science Foundation.

\section{References}

[1] Khayrutdinov R R and Lukash V E 1993 J. Comp. Physics 109193

[2] Jardin S C, Pomphrez N and DeLucia J 1986 Dynamic modelling of transport and positional control in a tokamak J. Computational Physics 66418

[3] Coutlis A, Bandyopadhyay I, Lister J B, Vyas P, Albanese R, Limebeer D J N, Villone F and Wainwright J P 1999 Nuclear Fusion 39663

[4] Hofmann F, Moret J-M and Ward D J 1998 Nuclear Fusion 381767

[5] Albanese R and Villone F 1998 Nuclear Fusion 38723

[6] Khayrutdinov R R, Lister J B, Lukash V E and Wainwright J P 2001 Comparing DINA code simulations with TCV experimental plasma equilibrium responses Plasma Physics and Controlled Fusion 43321

[7] Humphreys D A, Kellman A G, Khayrutdinov R R and Lukash V E 1995 Time-dependent simulation of DIII-D plasma evolution and control scenarios APS $\mathbf{4 0} 1191$ 
[8] Hofmann F et al 1997 Nuclear Fusion 37681

[9] Hofmann F, Favre A, Isoz P-F, Martin Y, Moret J-M and Nieswand C 2000 Nuclear Fusion 40 767

[10] Hofmann F and Tonetti G 1988 Nuclear Fusion 281871 


\section{TCV \#9478 $\mathrm{t}=0.3 \mathrm{~s}$}

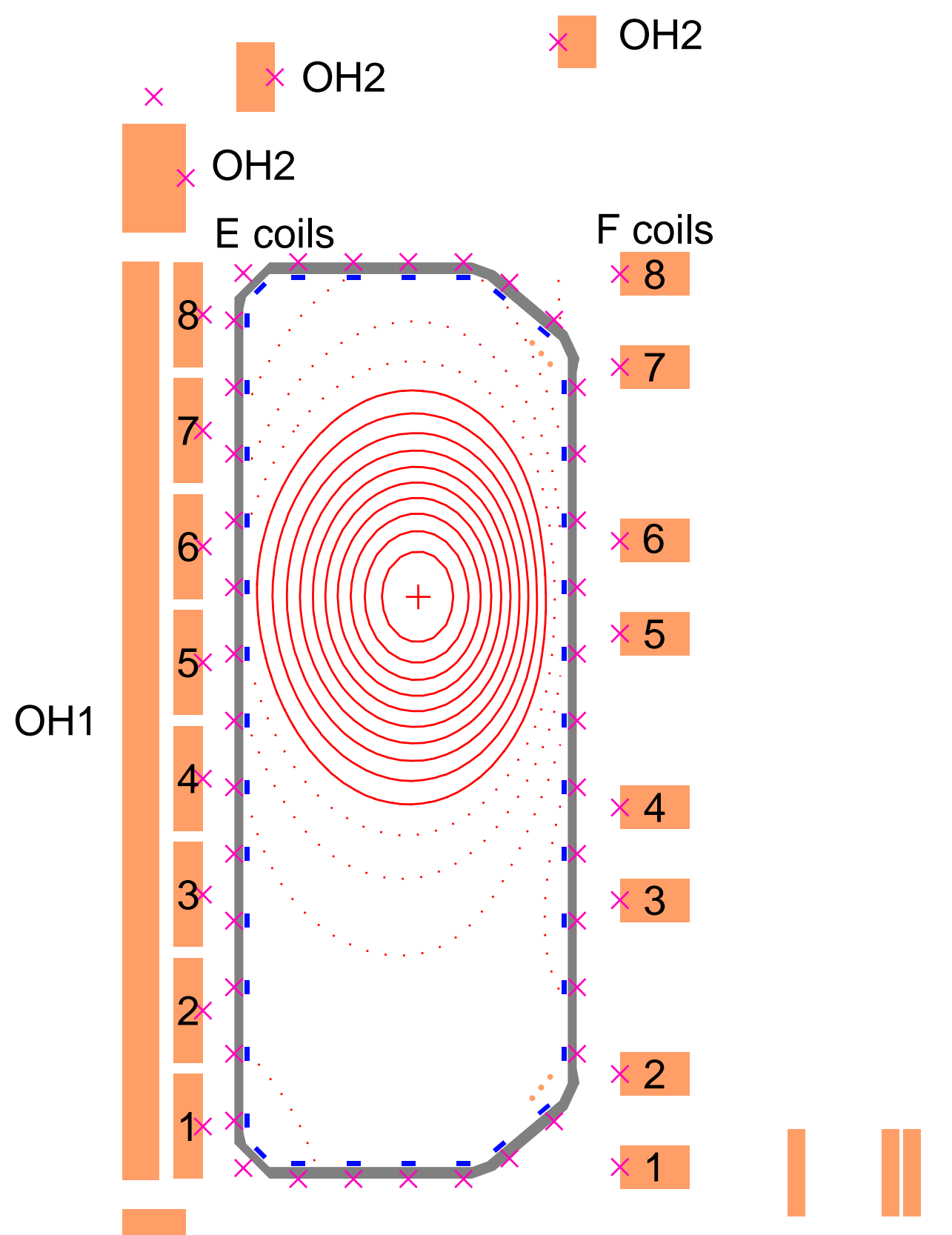

\section{$\mathrm{OH} 2$}

$\times \mathrm{OH} 2$

Figure 1. The TCV vacuum vessel, PF coils, poloidal field probes (marked '-' inside the tiles) and flux loops (marked 'x'). A modestly elongated plasma has a large volume available for a VDE before hitting the first wall 


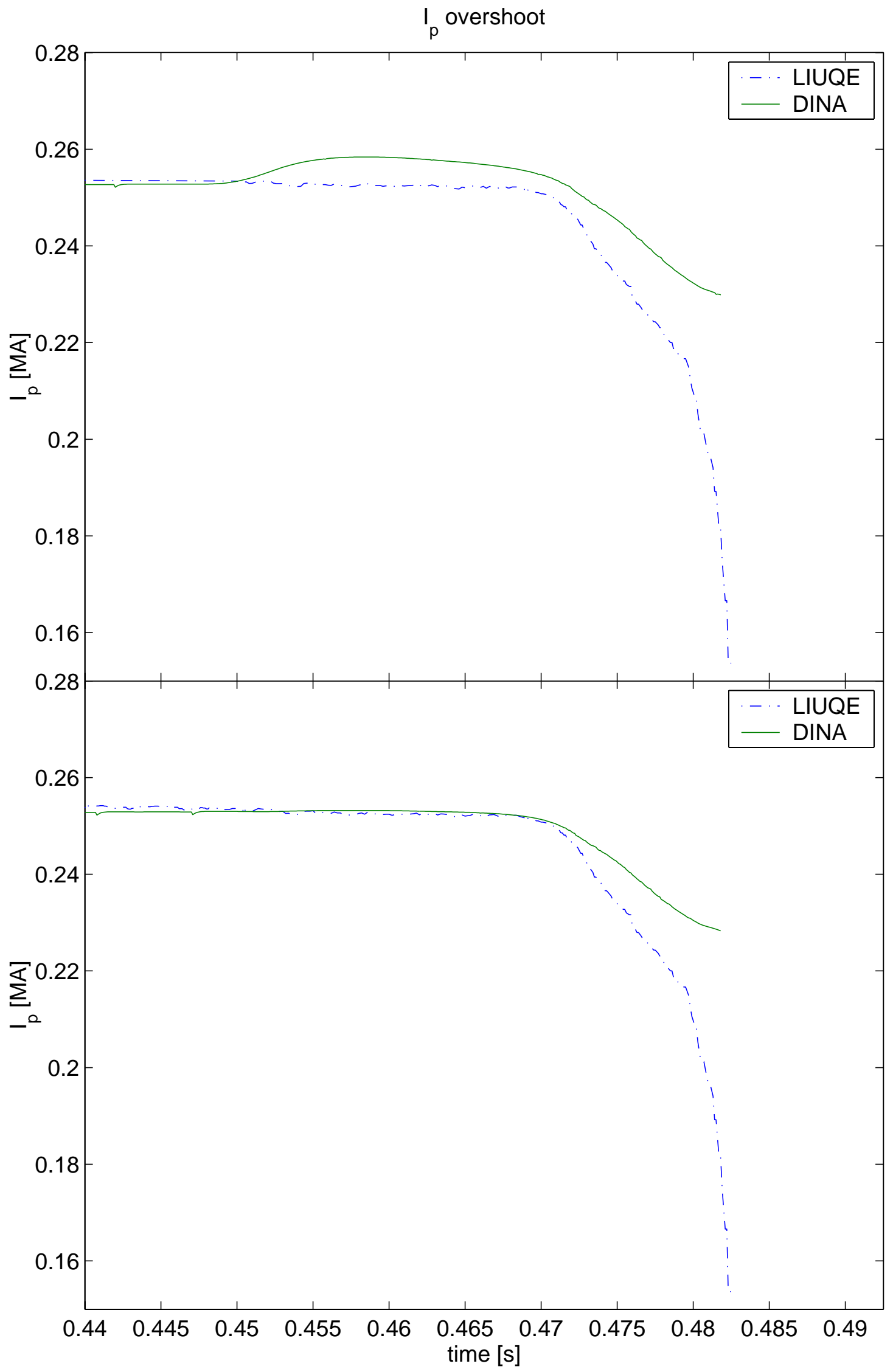

Figure 2. The figure on the top shows the plasma current Ip overshoot of DINA due to the large disturbance injected on the E1 coil only. The figure on the bottom shows no more overshoot because of the antisymmetric disturbance injection on coil E1 and E8. 


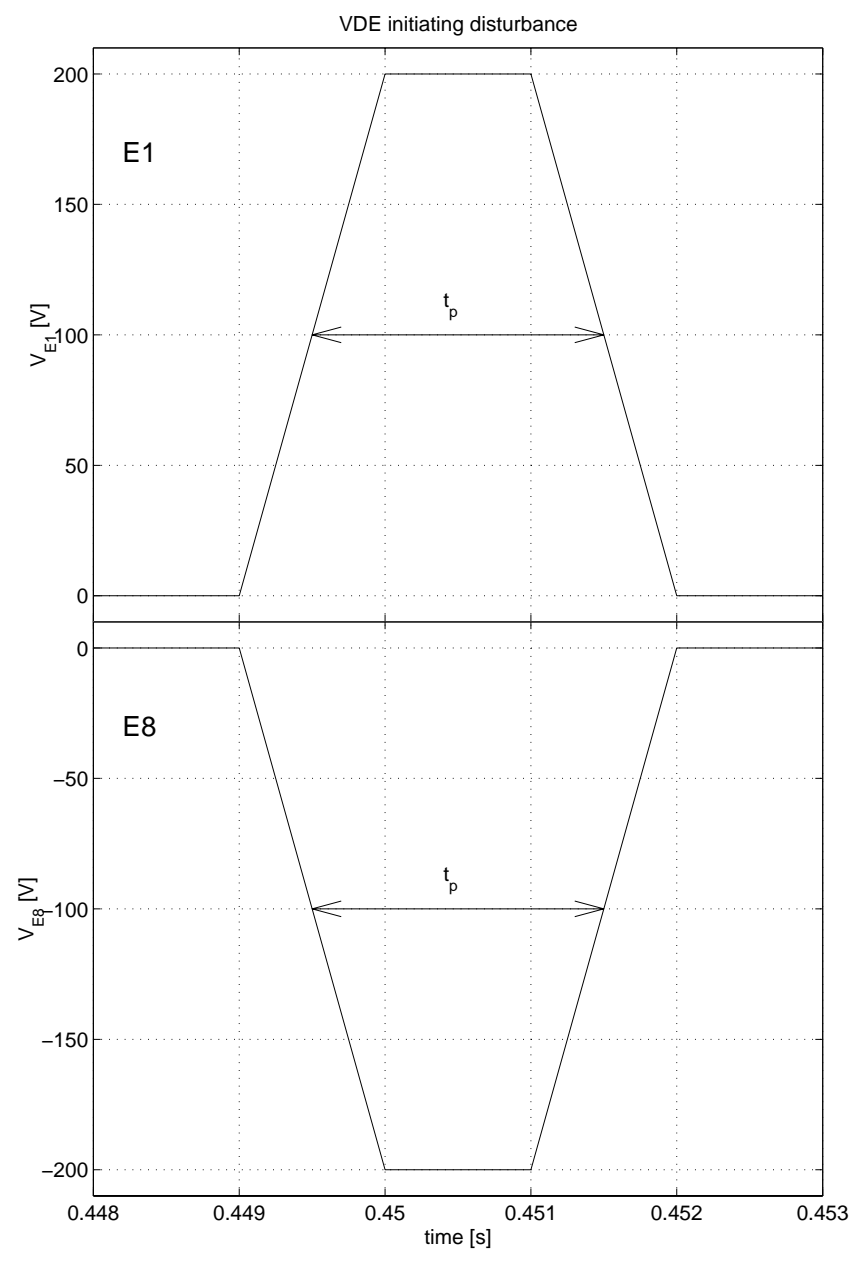

Figure 3. Disturbance shape initiating the VDE in DINA simulations. 


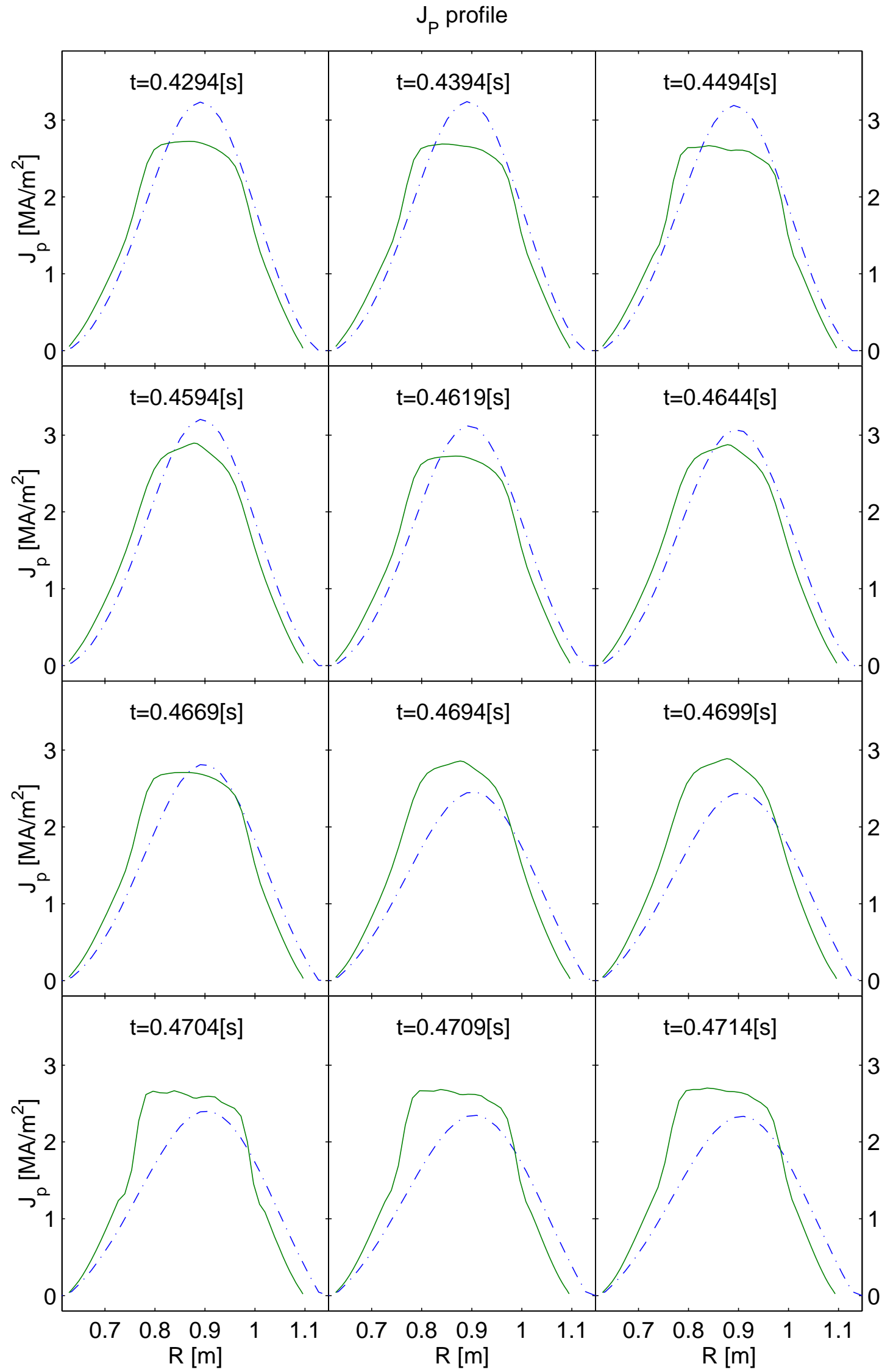

Figure 4. Plasma current density $J_{p}$ profile of discharge \#9487, estimated with LIUQE (dashed) and simulated with DINA (solid). 


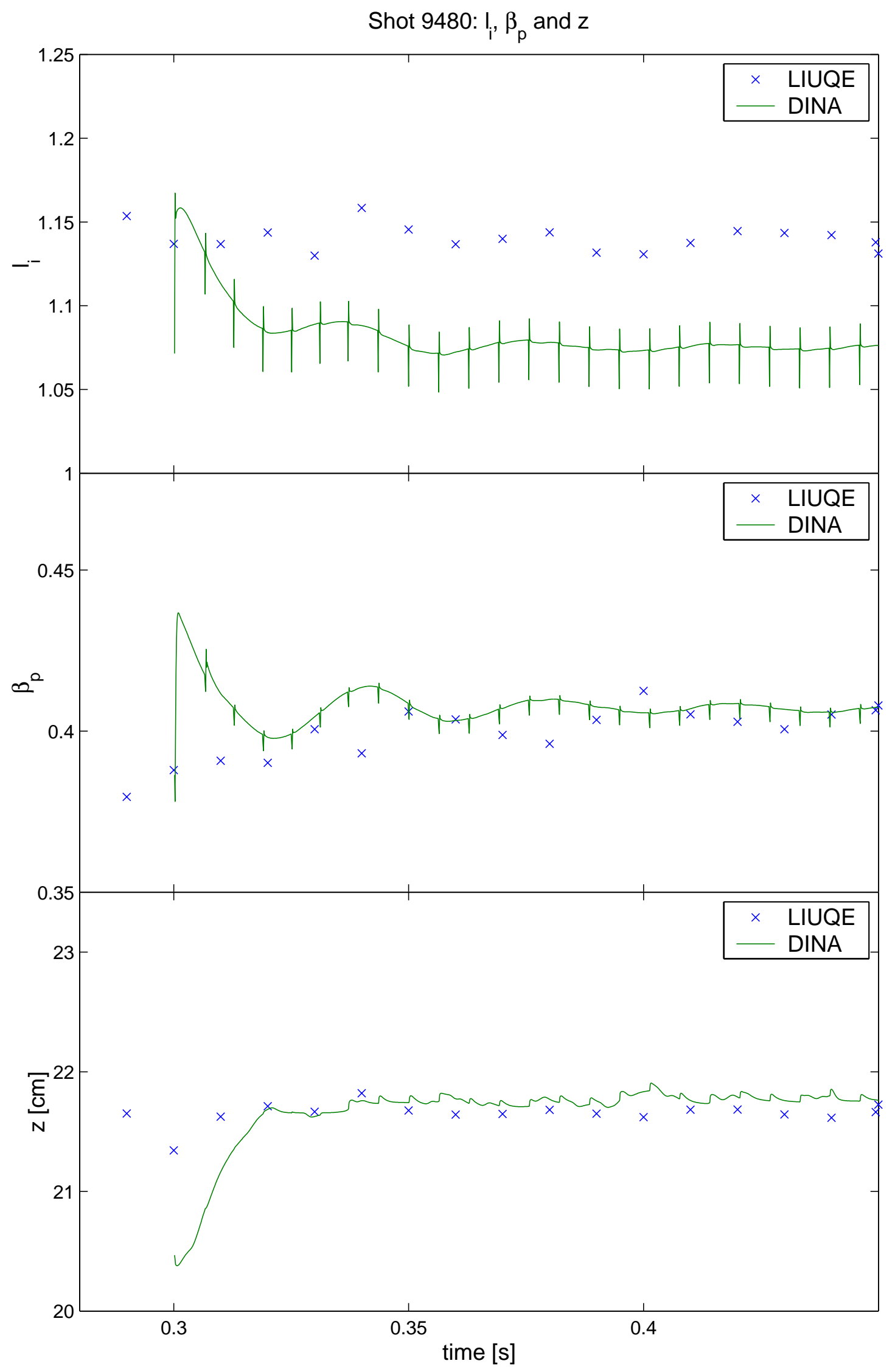

Figure 5. Discharge \#9480, showing a short transient of $l_{i}, \beta_{p}$ and the vertical position $z$ at the beginning of the DINA simulation. The perturbation peaks on the simulation are due to sawteeth. 


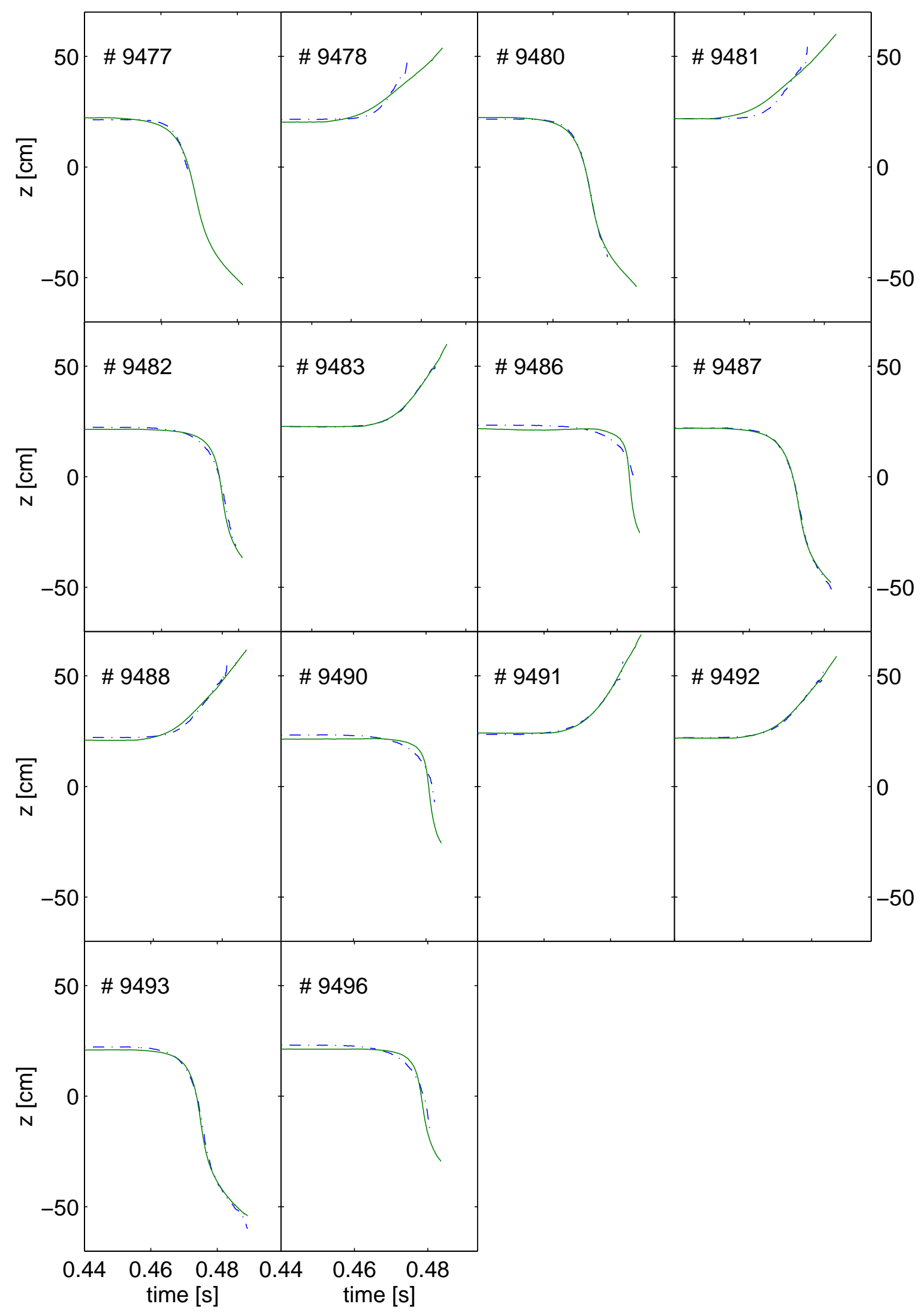

Figure 6. Comparison of the vertical displacement movement $z[\mathrm{~cm}]$. At $z= \pm 50 \mathrm{~cm}$ a full minor radius circular plasma hits the top or bottom tiles. (solid: DINA, dashed: LIUQE) 


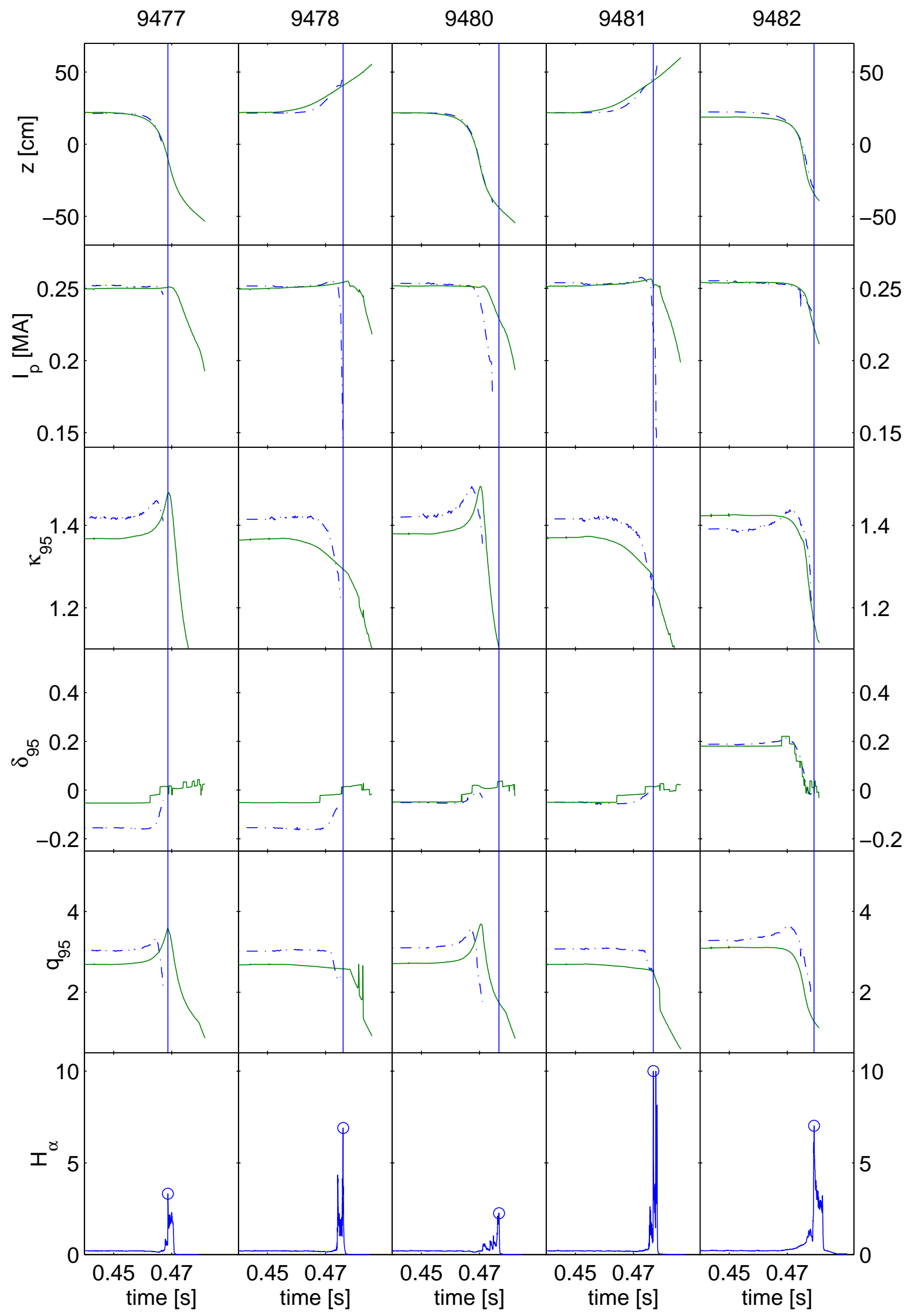

Figure 7. a) Comparison of the equilibrium parameters: vertical position $z$, plasma current $I_{p}$, elongation $\kappa_{95}$, triangularity $\delta_{95}$ and safety factor $q_{95}$. The peak of $H_{\alpha}$ indicates the disruption event, shown as a solid vertical line. (solid: DINA, dashed: LIUQE) 


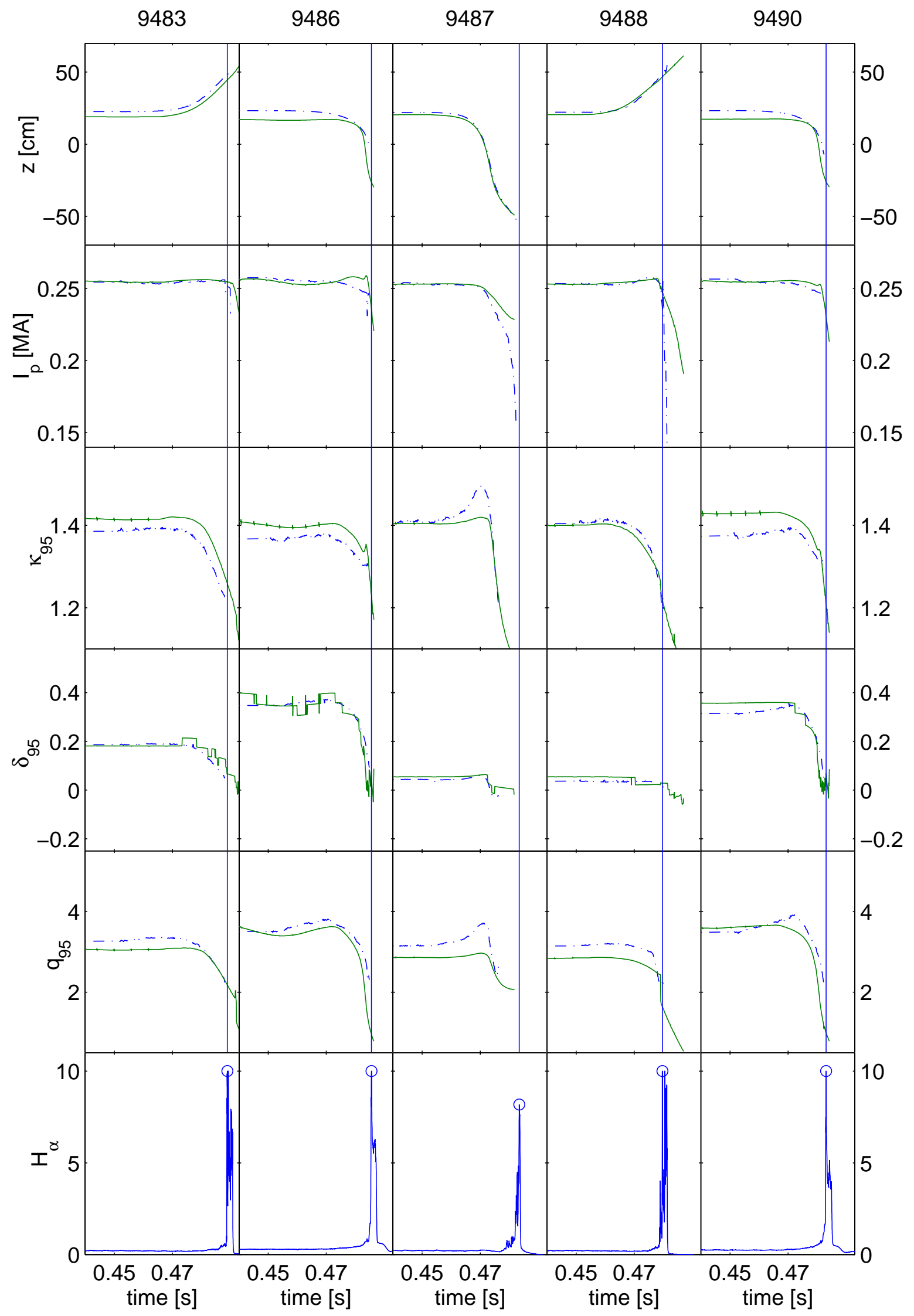

Figure 7. b) Comparison of the equilibrium parameters: vertical position $z$, plasma current $I_{p}$, elongation $\kappa_{95}$, triangularity $\delta_{95}$ and safety factor $q_{95}$. The peak of $H_{\alpha}$ indicates the disruption event, shown as a solid vertical line. (solid: DINA, dashed: LIUQE) 


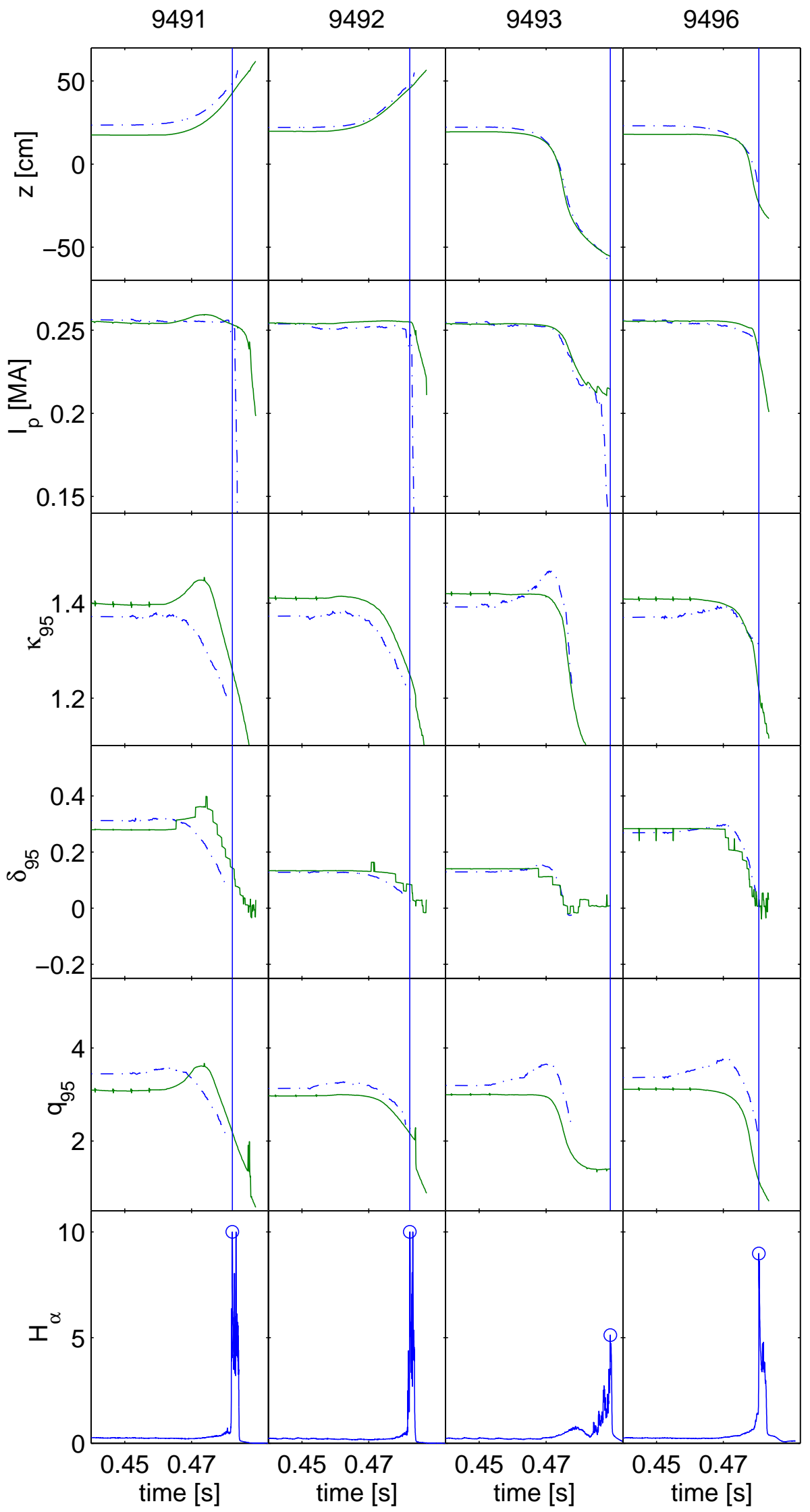

Figure 7. c) Comparison of the equilibrium parameters: vertical position $z$, plasma current $I_{p}$, elongation $\kappa_{95}$, triangularity $\delta_{95}$ and safety factor $q_{95}$. The peak of $H_{\alpha}$ indicates the disruption event, shown as a solid vertical line. (solid: DINA, dashed: LIUQE) 


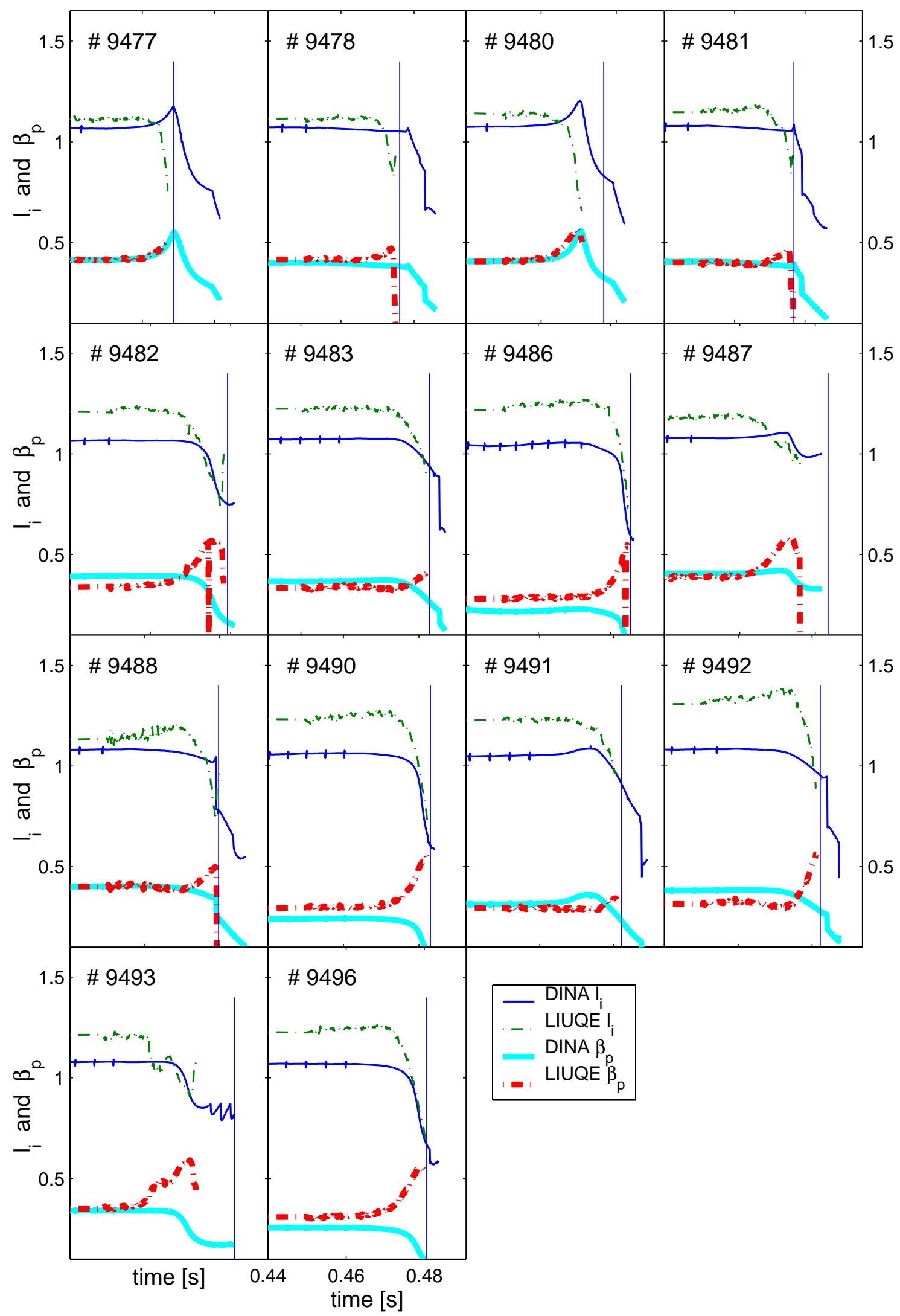

Figure 8. Comparison of $l_{i}$ and $\beta_{p}$. 


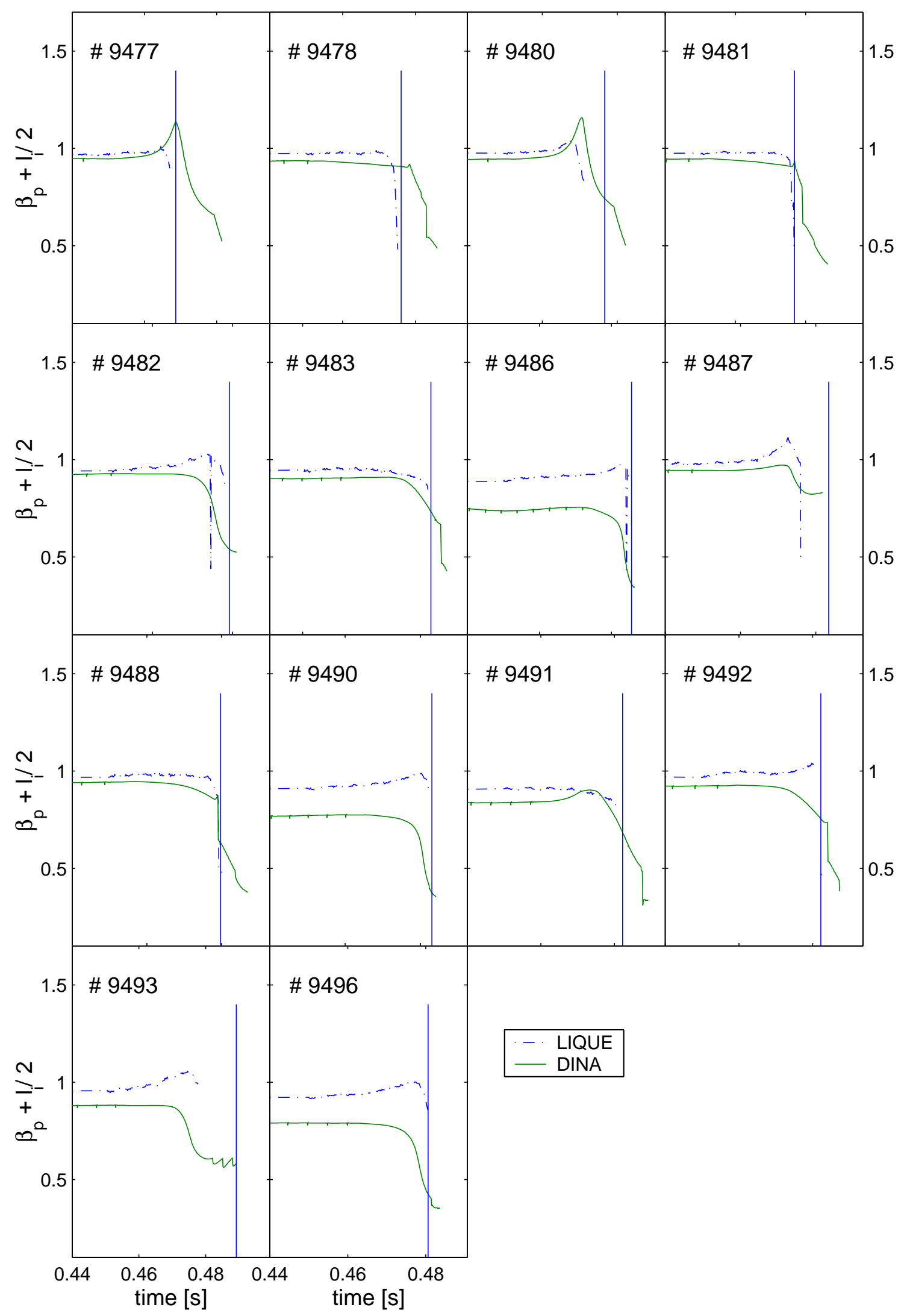

Figure 9. Comparison of $\beta_{p}+\frac{l_{i}}{2}$. 


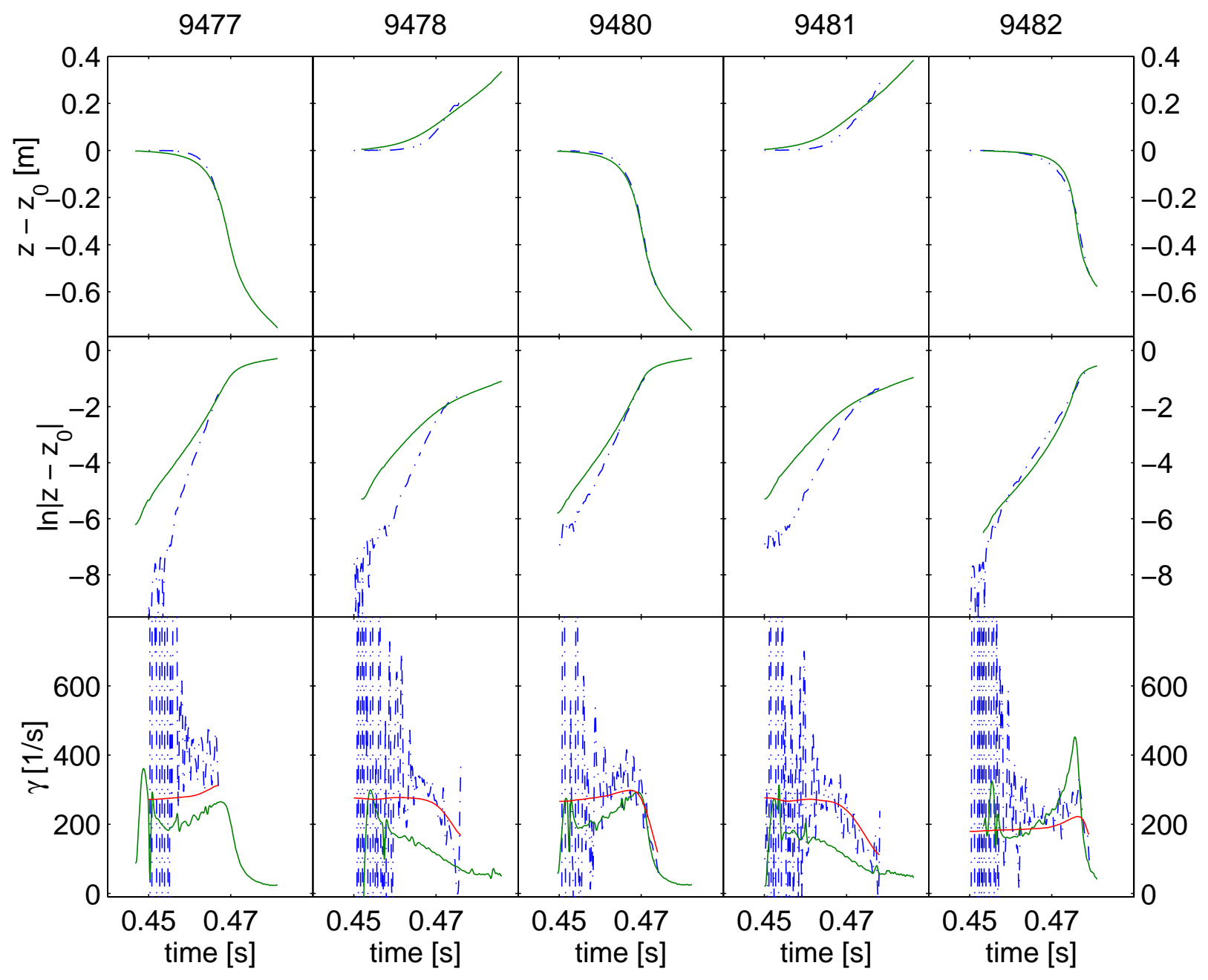

Figure 10. a) Computing growth rate of DINA (green solid) and LIUQE (blue dashed) and comparison with RZIP (red solid) growth rate. 


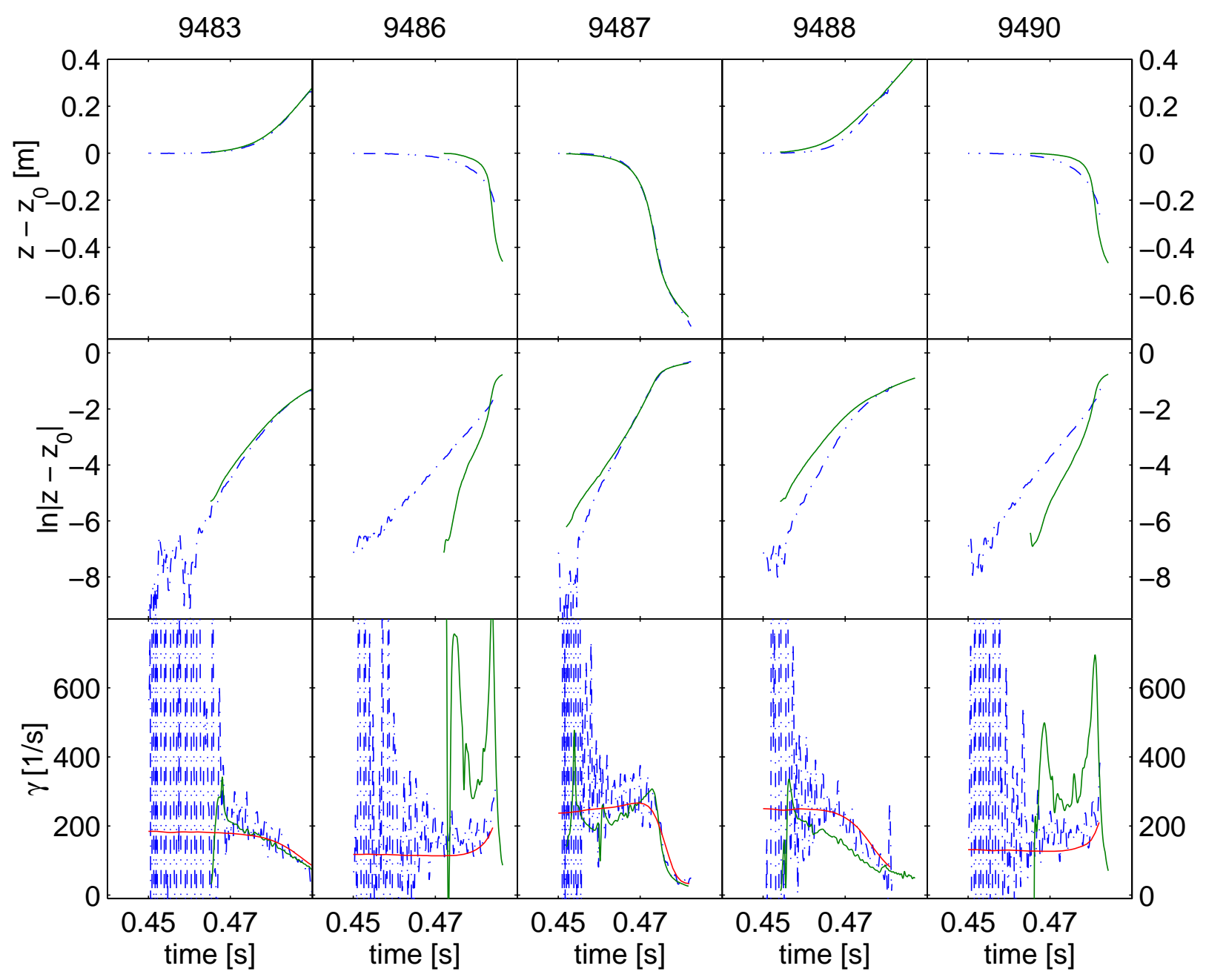

Figure 10. b) Computing growth rate of DINA (green solid) and LIUQE (blue dashed) and comparison with RZIP (red solid) growth rate. 


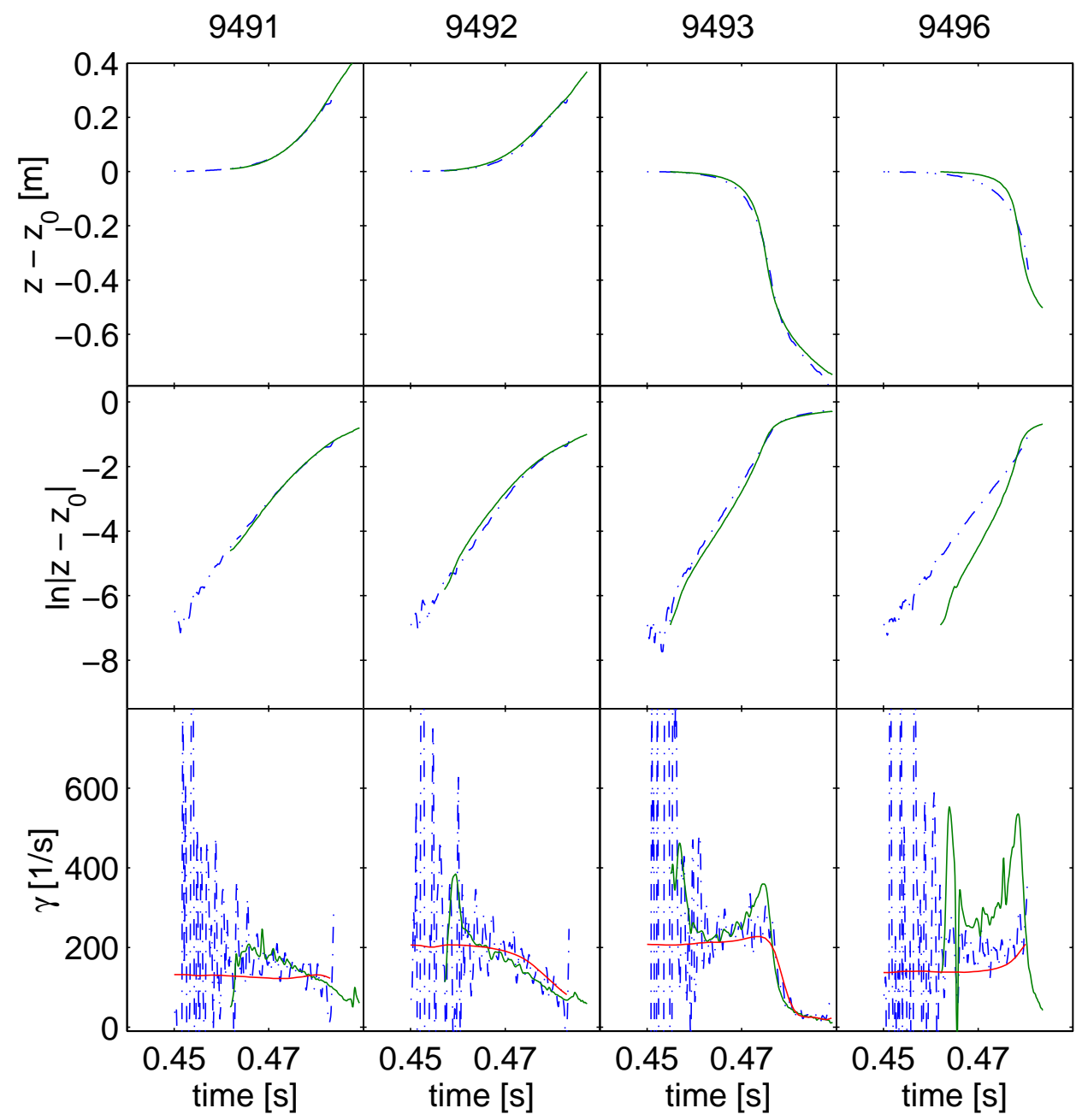

Figure 10. c) Computing growth rate of DINA (green solid) and LIUQE (blue dashed) and comparison with RZIP (red solid) growth rate. 


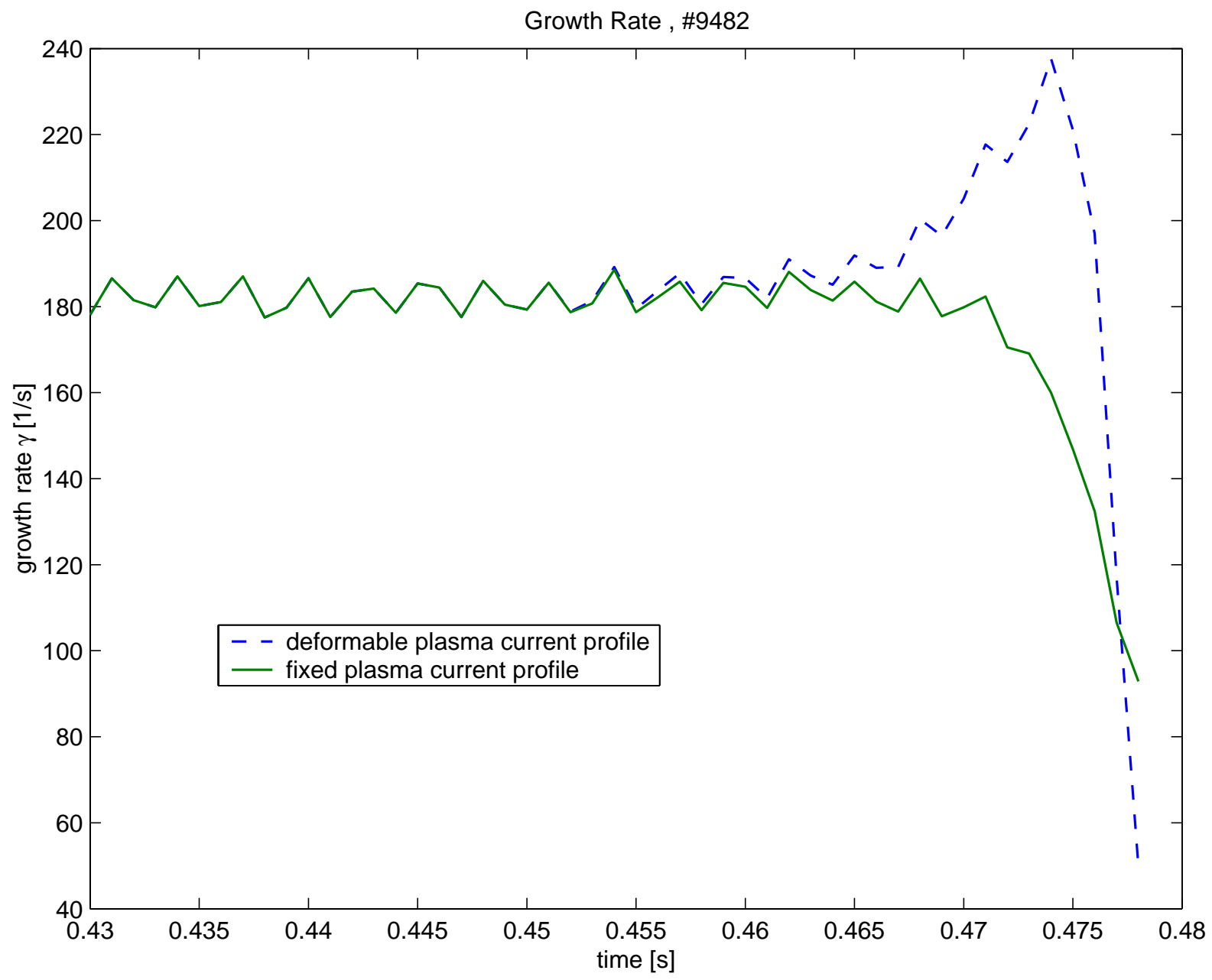

Figure 11. Evolution of the RZIP growth rate with a fixed plasma current profile (green solid) as against the 'normal' RZIP growth rate with a deformable reconstructed plasma current profile (blue dashed). The current profile of the fixed profile plot is taken at $t=0.43 \mathrm{~s}$. 


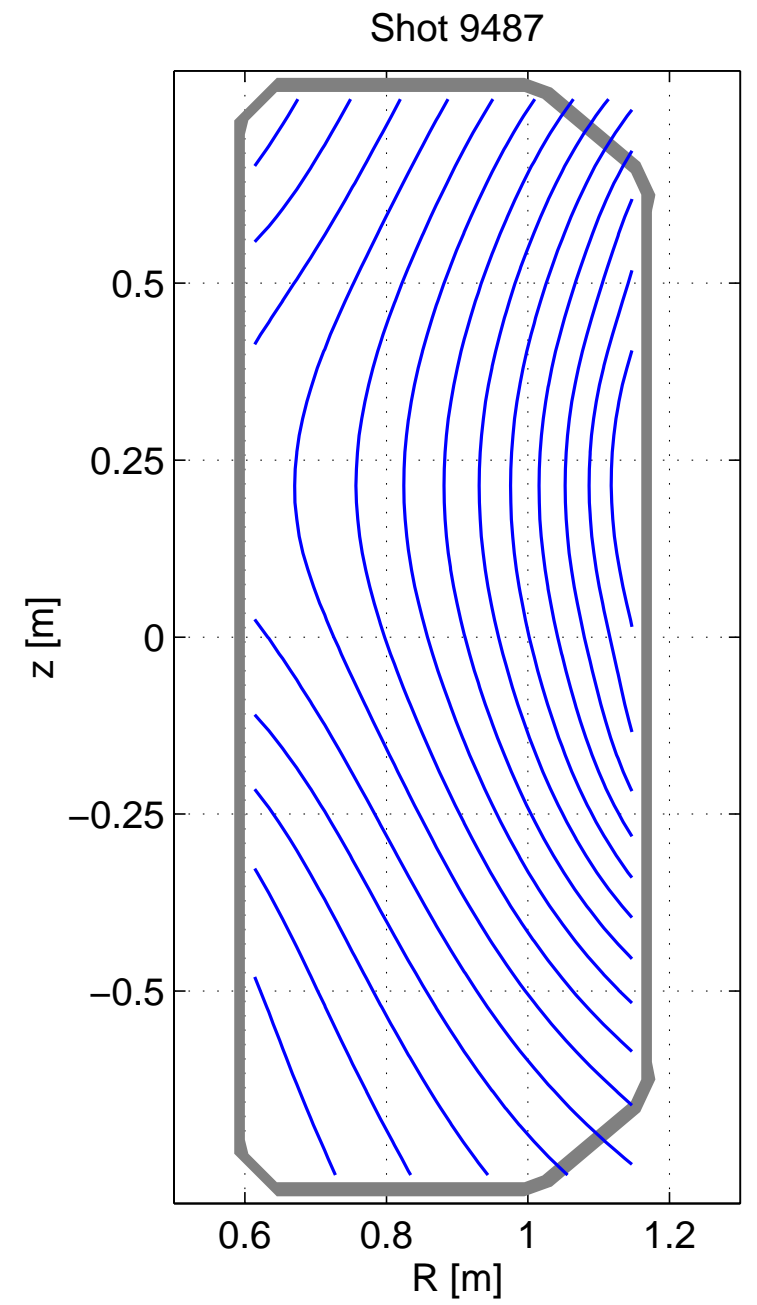

Figure 12. Spatial variation of the vacuum flux for the downward-going discharge \#9487. 

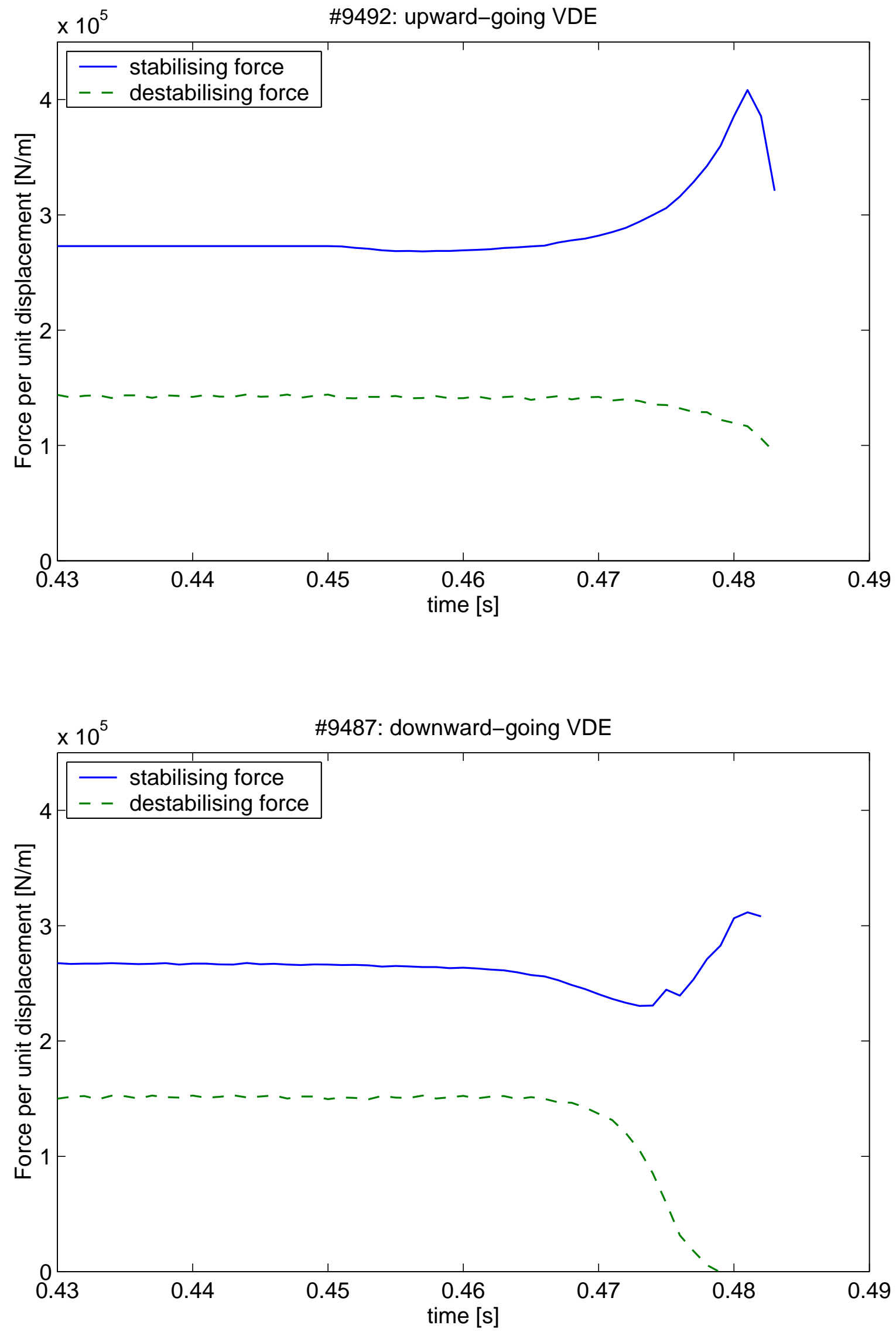

Figure 13. Evolution of the stabilising and destabilising forces per unit displacement for an upward-going VDE (top) and downward-going VDE (bottom). 